\title{
Assisted-Multicast Scheduling in Wireless Cognitive Mesh Networks
}

\author{
Hisham M. Almasaeid and Ahmed E. Kamal \\ Dept. of Electrical and Computer Eng., Iowa State University, Ames, IA 50011, USA \\ E-mail:\{hisham,kamal\}@iastate.edu
}

\begin{abstract}
In this work, we consider the multicast problem in a single cell in a cognitive mesh network. Due to the potential heterogeneity in channel availability among the members of a multicast group(s), the total multicast time could be longer due to transmitting the multicast data over multiple channels. We propose, in this work, an assisted multicast strategy with the objective of minimizing the total multicast time. This assistance is composed of two main activities, first, allowing the receivers in a multicast group to forward the data they have received to other members of the multicast group(s), and second, allowing the transmission of coded (bitwise XORed) packets so that receivers belonging to different multicast groups can decode and extract their data concurrently. We show, in this paper, that the proposed assistance paradigm achieves a considerable reduction in the total multicast time, which in turn increases the system throughput.
\end{abstract}

\section{INTRODUCTION}

Empowered by the technology of software-defined radios [1], cognitive radio networks have emerged as a solution for spectrum underutilization [2]. The new technology allows cognitive radio users, usually referred to as secondary users (SUs), to opportunistically utilize unused licensed spectrum provided that they vacate a channel once a licensed user, usually referred to as a primary user (PU), starts using it. As the channel availability is both time and location dependent, SUs may observe heterogeneous sets of idle channels. This heterogeneity raises a number of challenges to the operation of cognitive radio networks. Besides the channel sensing problem (or equivalently PU detection), transmission coordination is one of the essential and most challenging functionalities in cognitive radio networks. The channel sensing problem has been the focus of mainstream research in the area of cognitive radio networks. Cooperative sensing [3] and sensor networkaided-sensing [4], [5], where an infrastructure sensor network takes over the task of channel sensing, are two promising solutions for the PU detection problem. On the other hand, the most adopted solution for the transmission coordination problem is the use of a common control channel between all SUs on which the coordination takes place.

In this paper, we are interested in the multicast problem in wireless cognitive mesh networks [6]. The property of heterogenous channel availability in cognitive radio networks may cause the multicast process to take place over multiple channels, causing longer multicast periods and consequently lower throughput. We study, in this work, the problem of minimizing the total multicast time in a single cell of a cognitive mesh network by scheduling the multicast activity over both time and frequency and by also using the technique of network coding [7]. For this purpose, we propose a multicast mechanism that relies on three operations. The first operation is called assistance, in which some of the receiving members of a mutlicast group assist in the multicast process by forwarding the data to other members of their group (or other groups). The second operation is called overhearing, in which some receiving members of a multicast group overhear the data destined to another group. This operation has two advantages; it first enables the inter-group assistance (forwarding) between different multicast groups, and also enables the delivery of multiple packets to different groups at the same time by using the third operation; the codeword exchange operation. In the codeword exchange operation, coded packets (bitwise XORed packets), which we refer to as codewords, are used in the assistance operation so that members of different multicast groups can decode and extract their own data using packets they overheard from previous transmissions. Formal definitions of these operations will be given in Section III-A.

A very limited number of studies have addressed the multicast problem in cognitive radio networks. In [8], a multicast-tree construction protocol was proposed for mobile ad hoc networks. Video multicast in infrastructure cognitive radio networks was studied in [9]. The cognitive base station chooses the multicast channels opportunistically such that the collision probability with the primary system is minimized, and the unused spectrum is exploited as much as possible. In both works, there was no notion of assistance whether by multicast receivers or by the use of network coding.

The rest of this paper is organized as follows. The system model is presented in Section II. In Section III, we formally define the assisted multicast scheduling problem and present some motivational examples. Then, in Section IV, we elaborate on the problem complexity and propose ILP formulations for unassisted multicast scheduling and assisted multicast scheduling problems. A heuristic approach to solve the assisted multicast scheduling problem is proposed in Section V. We evaluate the performance of the proposed scheme in Section VI, and conclude in Section VII.

\section{SySTEM MOdEL}

We consider a wireless cognitive mesh network that consists of a number of mesh routers (MRs) connected over multiple hops to a gateway node(s) that provides access to the backbone network. Each MR manages a number of mesh client (MCs) 
forming a cell. The only way for a MC to access the backbone network is through its parent MR. This cognitive mesh network coexists with a primary (licensed) network(s) that utilizes a set of orthogonal channels $\mathcal{L}$. Secondary nodes (both MRs and MCs) can use any of these channels if the channel is vacant, either spatially or temporally. In this work, we assume that the channel availability at a node in the cognitive mesh network is quasi-static, i.e., does not change in a short period of time. Furthermore, MRs and MCs obtain information about channel availability by channel sensing [10], [11], [5], which is beyond the scope of this paper. Lastly, we assume that a free common control channel (CCC) exists for all the members of a cell in the cognitive mesh network to use for coordinating their communication.Data and control packets can be transmitted on separate radios, if nodes are equipped with multiple radios, or timely multiplexed over a single radio. In this work, we assume that one radio is used for data transmission per node ( $\mathrm{MC}$ or $\mathrm{MR}$ ), and that the data packet size is fixed.

For a particular cell managed by $\mathrm{MR} i$, let $\mathcal{L}_{i}$ be the set of channels that $i$ can use such that $\mathcal{L}_{i} \subseteq \mathcal{L}$, and $\mathcal{L}_{i} \neq \emptyset$. Also, let $\mathcal{A}_{i}$ be the set of MCs managed by MR $i$. Then for each $j \in \mathcal{A}_{i}, \mathcal{L}_{j} \subseteq \mathcal{L}_{i}$ is the set of channels that MC $j$ can use. We assume that all the members of a cell are in the same interference range, i.e., no two members of the cell can use the same channel concurrently. Once again, we are only concerned with the last stage of the multicast process, and that is from the MR to MCs that are members of a multicast group. Therefore, we do not study the multicast across multiple cells. We aim at minimizing the total multicast time (increasing throughput).

\section{Motivation And Problem Definition}

Before we formally define the assisted multicast problem, we would like to present an example that illustrates the motivation behind this work, and then give some definitions.

Example: Consider the network (a cell) in Figure 1. The figure shows two multicast groups: the white nodes form the group of MCs $\left\{n_{1}, n_{2}, n_{3}, n_{4}, n_{6}\right\}$ that should receive packet $a$, and the gray nodes form the group of MCs $\left\{n_{5}, n_{7}, n_{8}\right\}$ that should receive packet $b$. The set besides each MC represents the channels available to that MC. Node $n_{0}$ represents the MR of the cell, and it has all the five channels available. Table I, summarizes the basic idea of assisted multicast for the network in Figure 1. The first two rows show an optimal multicast schedule without any form of assistance, the first of the two shows the transmissions as (transmitter, packet, channel) tuples, and the second shows the receptions as (receivers, packet, channel) tuples. Similar pairs of rows are presented for three levels of assistance, each of which corresponds to exploiting an additional assistance operation. Columns in Table I correspond to time slots. As the table explains, under no form of assistance, the best the MR can do is 6 time slots. By adding intra-group assistance, i.e., allowing members of the same group to forward packets to each other, the total multicast time was reduced to 5 slots. By extending the assistance to inter-group (allowing receivers to assist members of other groups than theirs) the total time was further reduced to 4 time slots. The final step is to add network coding to the picture by allowing nodes (MRs or MCs) to exchange coded packets. This step reduced the total multicast time to 3 slots only. Note that MCs $n_{1}$ and $n_{6}$ (interested in packet $a$ ) have received $b$ in slot $T_{1}$, and MC $n_{5}$ (interested in packet $b$ ) have received $a$ in slot $T_{2}$. Therefore, all the three MCs will be able to decode the $a \oplus b$ packet and extract their own data. The multicast schedule using the three assistance mechanisms, intra-group assistance, inter-group assistance, and network coding, is presented in Figure 1. It is worth pointing out that a part of this scheduling problem that can highly affect the outcome is scheduling the overhearing opportunities especially if a MC has multiple transmissions to overhear from in a single time slot.

\section{A. Definitions}

We present in this subsection some necessary definitions.

Definition 3.1: Codeword: is a group of packets (could be a single packet) coded (bitwise XORed) into one packet.

Definition 3.2: Multicast period: is the number of time slots needed to deliver to all the members of each multicast group the data packet destined to that group.

Definition 3.3: Assistance operation: is the process of having an MC $i$ forward to an MC $j$ in slot $t$ a codeword that the latter can use, possibly with the codewords overheard in time $[0, t-1]$ by $j$, to extract the data destined to it. If $i$ and $j$ belong to the same group, then the operation is called Intra-group assistance. Otherwise, it is called Inter-group assistance.

Definition 3.4: Codeword exchange: is the process of allowing the exchange of codewords in the assistance operation.

Definition 3.5: Multicast schedule: is a schedule of the multicast activity over time and frequency. The schedule should determine for each member of a multicast group (including the MR) what to transmit/receive (packet), on what frequency (channel), and at what time (slot). The following are the feasibility conditions for a multicast schedule:

1) At a slot $t$, there can be at most one transmission per channel, and at most one transmission per node.

2) For an $\mathrm{MC}$ to transmit codeword $v$ at $t$, it must receive a set of codewords in $[0, t-1]$ sufficient to construct $v$.

Then, the assisted mutlicast scheduling (AMS) problem in cognitive mesh networks is defined as follows:

Definition 3.6: AMS problem in cognitive mesh networks: Given $M$ multicast groups $\left\{\mathcal{G}_{1}, \cdots, \mathcal{G}_{M}\right\}$ managed by MR $i$ such that $\mathcal{G}=\bigcup_{j=1}^{M} \mathcal{G}_{j} \subseteq \mathcal{A}_{i}$, find a feasible multicast schedule, with both assistance and codeword exchange operations enabled, that results in the minimum multicast period.

\section{PRoblem COMPlexity And Formulation}

In this section, we study the complexity of the AMS problem and propose two integer linear program (ILP) formulations for the case of unassisted multicast, and the case of single multicast group with intra-group assistance.

\section{A. Single Multicast Group}

We first consider the case where only one multicast group, denoted as $\mathcal{G}$, exists in a cell managed by MR $n_{0}$. Therefore, the only possible form of assistance is the intra-group 

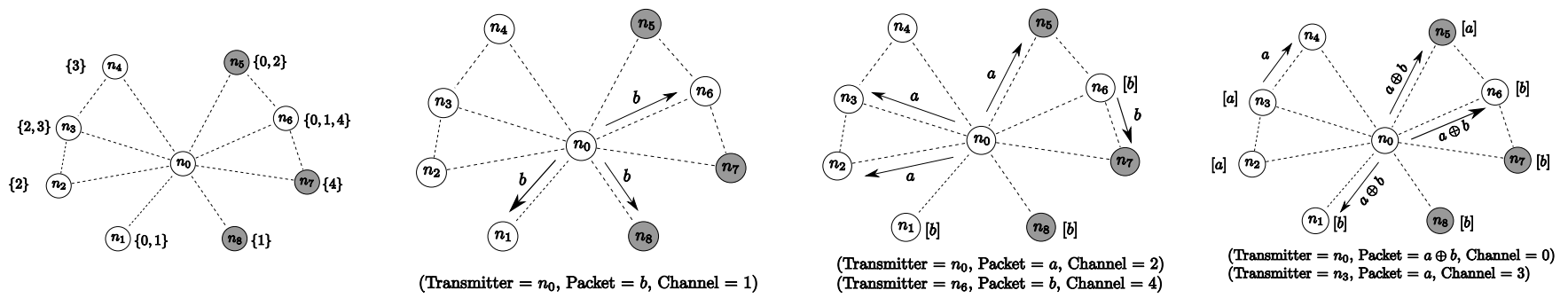

Fig. 1. An example that shows the benefit of using assisted multicast in reducing the total multicast period.

TABLE I

ENHANCING THROUGHPUT BY INTRODUCING DIFFERENT ASSISTANCE MECHANISMS.

\begin{tabular}{|c|c|c|c|c|c|c|c|}
\hline Scenario & $T x / R x$ & $T_{1}$ & $T_{2}$ & $T_{3}$ & $T_{4}$ & $T_{5}$ & $T_{6}$ \\
\hline \multirow[t]{2}{*}{ Unassisted multicast } & $T x$ & $\left(n_{0}, a, 0\right)$ & $\left(n_{0}, a, 2\right)$ & $\left(n_{0}, a, 3\right)$ & $\left(n_{0}, b, 0\right)$ & $\left(n_{0}, b, 4\right)$ & $\left(n_{0}, b, 1\right)$ \\
\hline & $R x$ & $\left(\left\{n_{1}, n_{6}\right\}, a, 0\right)$ & $\left(\left\{n_{2}, n_{3}\right\}, a, 2\right)$ & $\left(n_{4}, a, 3\right)$ & $\left(n_{5}, b, 0\right)$ & $\left(n_{7}, b, 4\right)$ & $\left(n_{8}, b, 1\right)$ \\
\hline \multirow[t]{2}{*}{ Intra-group assis. } & $T x$ & $\left(n_{0}, a, 0\right)$ & $\left(n_{0}, a, 2\right)$ & $\left(n_{3}, a, 3\right),\left(n_{0}, b, 0\right)$ & $\left(n_{0}, b, 4\right)$ & $\left(n_{0}, b, 1\right)$ & - \\
\hline & $R x$ & $\left(\left\{n_{1}, n_{6}\right\}, a, 0\right)$ & $\left(\left\{n_{2}, n_{3}\right\}, a, 2\right)$ & $\left(n_{4}, a, 3\right),\left(n_{5}, b, 0\right)$ & $\left(n_{7}, b, 4\right)$ & $\left(n_{8}, b, 1\right)$ & - \\
\hline \multirow[t]{2}{*}{ Inter-group assis. } & $T x$ & $\left(n_{0}, b, 1\right)$ & $\left(n_{0}, a, 2\right),\left(n_{6}, b, 4\right)$ & $\left(n_{3}, a, 3\right),\left(n_{0}, b, 0\right)$ & $\left(n_{0}, a, 0\right)$ & - & - \\
\hline & $R x$ & $\left(\left\{n_{8}, n_{6}\right\}, b, 1\right)$ & $\begin{array}{l}\left(\left\{n_{2}, n_{3}\right\}, a, 2\right) \\
\left(n_{7}, b, 4\right)\end{array}$ & $\left(n_{4}, a, 3\right),\left(n_{5}, b, 0\right)$ & $\left(\left\{n_{1}, n_{6}\right\}, a, 0\right)$ & - & - \\
\hline \multirow[t]{2}{*}{ Codeword exchange } & $T x$ & $\left(n_{0}, b, 1\right)$ & $\left(n_{0}, a, 2\right),\left(n_{6}, b, 4\right)$ & $\left(n_{3}, a, 3\right),\left(n_{0}, a \oplus b, 0\right)$ & - & - & - \\
\hline & $R x$ & $\left(\left\{n_{1}, n_{6}, n_{8}\right\}, b, 1\right)$ & $\begin{array}{l}\left(\left\{n_{2}, n_{3}, n_{5}\right\}, a, 2\right), \\
\left(n_{7}, b, 4\right)\end{array}$ & $\begin{array}{l}\left(n_{4}, a, 3\right) \\
\left(\left\{n_{1}, n_{5}, n_{6}\right\}, a \oplus b, 0\right)\end{array}$ & - & - & - \\
\hline
\end{tabular}

assistance between the members of $\mathcal{G}$. To understand the complexity of the "AMS for a single group" problem, let us study that of the normal, unassisted, mutlicast scheduling problem as the latter is a special case of the former.

Definition 4.1: Unassisted Multicast scheduling for a single group (UMS-Single): given a multicast group $\mathcal{G}$ managed by $\operatorname{MR} n_{0}$ and the set of available channels at each MC $i \in \mathcal{G}$ and $n_{0}$. Find a multicast schedule that results in the minimum multicast period given that $n_{0}$ is the only transmitter.

Theorem 4.1: The UMS-Single problem is NP-hard.

Proof: a reduction from the set-cover problem can be easily drawn. The set cover problem has, as input, a universe $\mathcal{U}$ and a group of subsets $\mathcal{S}=\left\{S_{1}, \cdots, S_{M}\right\}$, and the objective is to find the minimum number of subsets that cover the universe $\mathcal{U}$, i.e., Minimize $|\mathcal{C}|: \mathcal{C} \subseteq \mathcal{S}, \bigcup_{c \in \mathcal{C}} c=\mathcal{U}$. To map an instance of the set-cover problem into an instance of the UMSsingle, we do the following:

- Create a hypothetical node $n$ and mark it as the MR.

- For each member $u \in \mathcal{U}$ in the set-cover problem, create an MC $u$ in the UMS-Single problem and extend an edge between $u$ and $n$.

- Map each subset $S_{k}$ in the set-cover problem into a channel $k$ in UMS-Single problem. Then, make channel $k$ available to every MC $u$ iff $u \in S_{k}$.

- Make all channels available to the MR $n$.

Note that in the UMS-Single problem, MR $n$ is the only transmitter and it transmits on one channel at each time slot. Also, note that any solution that has the MR transmits on the same channel in different time slots is not optimal, because the exact same set of MCs will receive the packet in both transmissions. Therefore, the minimum number of time slots to deliver the multicast packet to all MCs maps directly, by construction, to the minimum number of sets that can cover $\mathcal{U}$. In the other direction, the minimum number of subsets that cover the universe maps, also by construction, to the minimum number of time slots (because we use one channel per slot) needed to deliver the multicast packet.

Before we present the ILP formulations, we need to present some notations:

- $\mathcal{G}$ is the only multicast group in the cell managed by MR $n_{0}$ (excluding $n_{0}$ ) such that $\mathcal{G} \subseteq \mathcal{A}_{0}$.

- $\mathcal{N}(i)$ is the set of nodes in $\mathcal{G} \cup\{0\} \backslash\{i\}$ that can reach node $i$ on at least one channel.

- $\mathcal{L}$ is the set of all channels available to the members of the multicast group $\left(\mathcal{L}_{i} \subseteq \mathcal{L}_{0} \forall i \in \mathcal{A}_{0}\right)$.

- $T$ is the maximum number of time slots needed to deliver the multicast packet to all the members of a multicast group. $T$ is upper bounded by $\min \{|\mathcal{L}|,|\mathcal{G}|\}$, and for simplicity we set $T=\min \{|\mathcal{L}|,|\mathcal{G}|\}$.

- $\nu^{t}$ is a binary variable that is set to 1 iff a transmission exists in slot $t$ on any of the channels in $\mathcal{L}$.

- $y_{i, k}^{t}$ is a binary variable that, if set to 1 , indicates that node $i$ transmits the packet on channel $k$ at slot $t$.

The UMS-Single problem is now formulated as an ILP in Algorithm 1. The objective function is to minimize the total number of used (transmitted in) time slots. Constraint (2) guarantees that each MC will receive the data by forcing the MR to transmit on at least one of the channels available to that MC. One transmission per slot is forced by (3).

The ILP presented in Algorithm 1 can be modified to formulate the AMS problem with intra-group assistance for a single multicast group. We just need to allow MCs to forward the data they receive to their neighbors. The ILP 


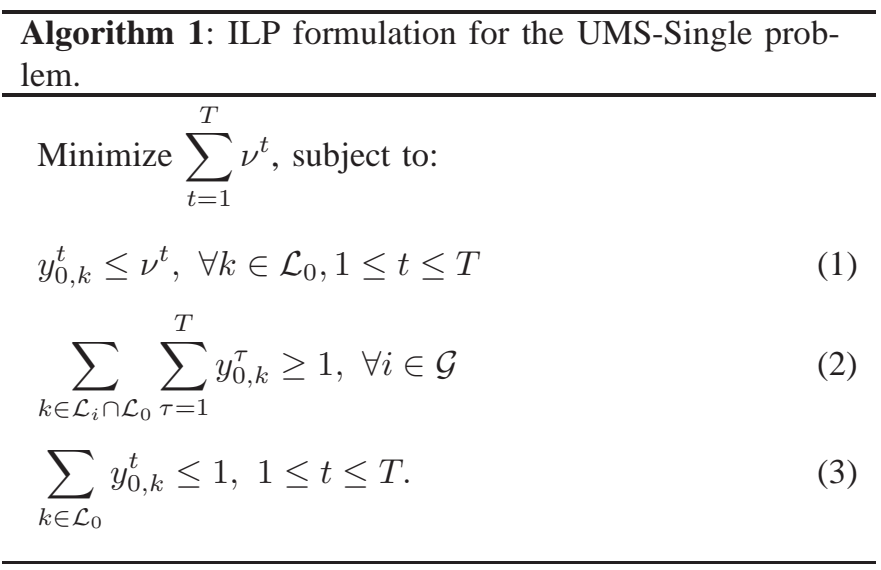

formulation for the AMS problem with a single multicast group is presented in Algorithm 2. Using constraint (5), we forbid MCs from transmitting in the first time slot as they have not received the multicast packet yet. Constraint (6) guarantees that no $\mathrm{MC}$ transmits on any channel at slot $t$ before it receives the packet from at least one neighbor, on a channel common between the two, in $[1, t-1]$. We guarantee the delivery of the multicast packet to each MC by constraint (7). Constraints (8) and (9) guarantee one transmission per channel and one transmission per node in each time slot respectively.

\section{B. Multiple Multicast Groups}

Apparently, the problem of AMS with multiple groups is at least as hard as the AMS with a single group, which is NP-hard. Furthermore, the ILP formulation of the AMS problem with multiple groups is very complicated because of the codeword exchange operation. Specifically, an MC cannot transmit a codeword $v$ at time $t$ unless it receives a set of codewords sufficient to construct $v$. To embed this fact in the constraint of the ILP, we need to take into consideration all combinations of native multicast packets which will increase the number of variables and the constraints exponentially. Moreover, the constraint which ensures that each $\mathrm{MC}$ receives its multicast packet is also more complicated. Instead of a unique packet that satisfies the constraint in the case of a single group, a group of decodable codewords can satisfy the delivery constraint in the case of multiple groups with the codeword exchange operation. This requires us to take into consideration all the combinations of decodable codewords from which an MC can extract its packet. This will also increase the number of constraints exponentially. Therefore, we do not propose an ILP formulation for the AMS problem with multiple groups.

\section{Heuristic Solution For the AMS PROBlem}

In this section, we propose a heuristic algorithm to solve the AMS problem in cognitive mesh networks. The algorithm is greedy-based in the sense that we deal with each slot independently and try to make the optimal decision at this slot. However, finding this optimal decision is not easy. In fact, it can be shown that for the case of a single multicast group, scheduling the transmissions of the MR and covered

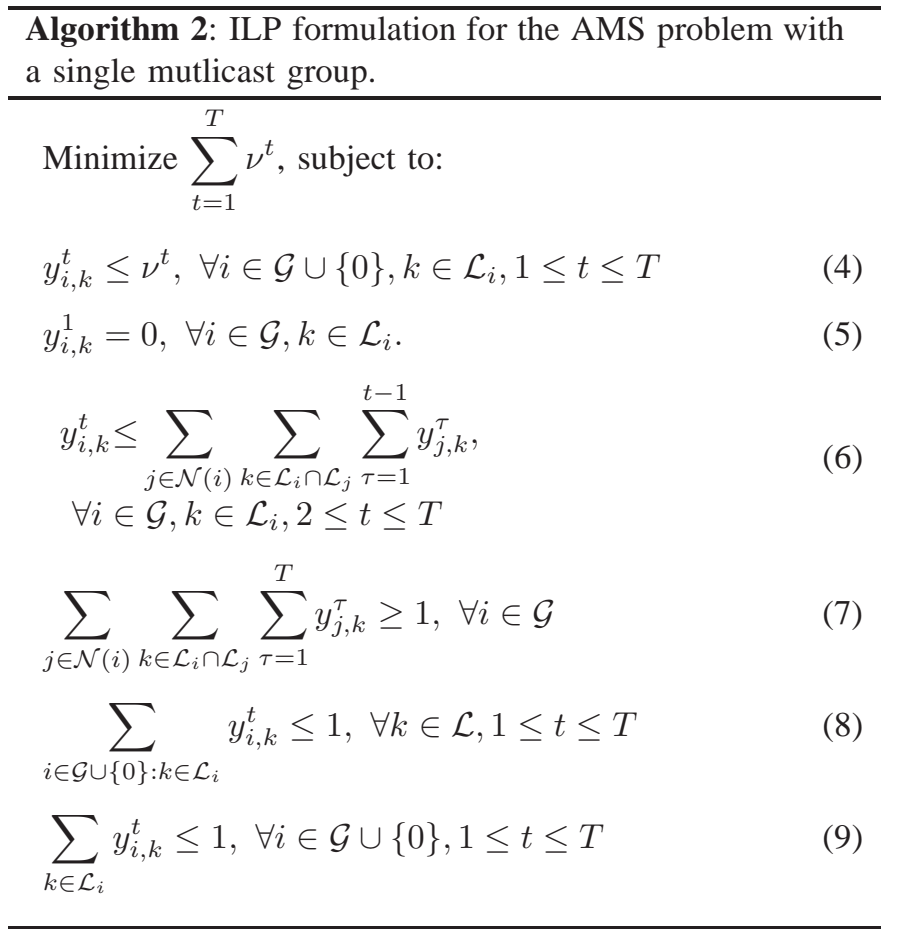

MCs, those which have received the packet in $[0, t-1]$, at a slot $t$ such that the packet is delivered to the maximum number of uncovered MCs is NP-hard (the proof is omitted due to lack of space). Therefore, we divide the scheduling task at a single time slot into three phases. (1) Scheduling the MR transmission (channel and codeword). (2) Scheduling the assistance operation (channel and codeword). (3) Scheduling overhearing opportunities. Note that all these operations are scheduled over frequency channels only and not over time slots. Before we present the details of each of the three phases, we need to provide some terminology.

Let $V_{i}$ be the set of overheard codewords by MC $i$ up until the current time slot. Also, let $p(i)$ be the multicast packet destined to multicast group to which $\mathrm{MC} i$ belongs. Then, for a MC $i$ that has not yet received $p(i)$, and has overheard the set of codewords $V_{i}$, the set of useful codewords (those that $i$ can decode and extract $p(i)$ from) can be determined as follows. Define $\mathcal{V}_{i}$ as the set of all combinations of the codewords in $V_{i}$, i.e., $\left|\mathcal{V}_{i}\right|=2^{\left|V_{i}\right|}-1$. Then, $p(i) \oplus \mathcal{V}_{i}^{k}$ is a useful codeword for MC $i$, where $\mathcal{V}_{i}^{k}$ denotes the $k^{t h}$ combination (codeword) of $\mathcal{V}_{i}$, for all $1 \leq k \leq\left|\mathcal{V}_{i}\right|$.

Let $\mathcal{G}_{c} \subseteq \mathcal{G}$ denotes the set of MCs that have received their multicast packets. Then, the best (codeword, channel) schedule for the MR, $n_{0}$, in a given slot is found as follows:

$\left(v^{*}, k^{*}\right)=\underset{\left(v \in \mathcal{V}, k \in \mathcal{L}_{0}\right)}{\operatorname{argmax}} \sum_{i \in \mathcal{G} \backslash \mathcal{G}_{c}} \begin{cases}1 & \text { if } v \oplus p(i) \in \mathcal{V}_{i}, k \in \mathcal{L}_{i} \\ 0 & \text { otherwise }\end{cases}$

where $\mathcal{V}$ is defined as,

$\mathcal{V}=\bigcup_{i \in \mathcal{G} \backslash \mathcal{G}_{c}} \mathcal{V}_{i}$ 
Equation (10) basically finds, for the MR, the (codeword, channel) pair that serves the maximum number of unserved MCs at a particular time slot. The same approach is used for the second phase, namely, scheduling the assistance operation. For an MC $i$ that can participate in the assistance process, its optimal (codeword, channel) is found as follows:

$$
\left(v_{i}^{*}, k_{i}^{*}\right)=\underset{\left(v \in \mathcal{V}_{i}, k \in \mathcal{L}_{i} / \mathcal{K}\right)}{\operatorname{argmax}} \sum_{w \in \mathcal{G} \backslash \mathcal{G}_{c}}\left\{\begin{array}{c}
1 \quad \begin{array}{c}
\text { if } v \oplus p(w) \in \mathcal{V}_{w}, \\
k \in \mathcal{L}_{w}, w \in \mathcal{N}(i) \\
0 \\
\text { otherwise }
\end{array}
\end{array}\right.
$$

where $\mathcal{K}$ is the set of used channels in the current slot, i.e., the set of channels that MC $i$ cannot transmit on. The last phase is to schedule the overhearing operation for MCs that are not participating in the assistance operation. The basic idea is for a MC to overhear the codeword that is useful to the maximum number of its neighbors. Let $\mathcal{T}$ be the set of all transmissions in the current time slot made by the MR and the MCs participating in the assistance operation and represented as (codeword, channel) pairs. Then, for a non-transmitting MC $i$, the best (codeword, channel) for overhearing is:

$$
\left(v_{i}^{*}, k_{i}^{*}\right)=\underset{(v, k) \in \mathcal{T}}{\operatorname{argmax}} \sum_{w \in \mathcal{G} \backslash \mathcal{G}_{c}}\left\{\begin{array}{l}
1 \quad \text { if } v \in \bigcup^{\left|\mathcal{V}_{w}\right|} \mathcal{V}_{w}^{z} \oplus p(w), \\
k \in \mathcal{L}_{w}^{z=1} \mathcal{L}_{i}, w \in \mathcal{N}(i) \\
0 \text { otherwise }
\end{array}\right.
$$

The AMS heuristic approach, denoted HAMS, is outlined in Algorithm 3. The first phase, i.e., scheduling the MR transmission, is expressed by lines $[6-12]$. The phase of scheduling the assistance operation is expressed by lines [14-27]. Finally, the phase of scheduling overhearing opportunities is expressed in the loop starting at line 28 . Note that for the case of $M$ groups, $\mathcal{N}(i)$ is defined as the set of nodes in $\left(\bigcup_{j=1}^{M} \mathcal{G}_{j}\right) \cup\{0\} \backslash\{i\}$ that can reach $\mathrm{MC} i$ in at least one channel.

\section{PERformance Evaluation}

In this section, we evaluate the performance of the proposed assistance mechanism for the multicast scheduling problem in wireless cognitive mesh networks. We vary the number of MCs from 5 to 50 distributed uniformly at random in a square area of $500 \mathrm{~m} \times 500 \mathrm{~m}$. The MR's location is fixed at the center of the squared area. All nodes (MCs and the MR) are assumed to have the same communication radius of $\sqrt{2} \times \frac{500}{2}=353.55 \mathrm{~m}$ over all channels. We vary the number of multicast groups $M$ between 1, 3, 4, and 5. Each MC is assigned to any of the $M$ groups uniformly at random. Lastly, we have the number of channels $K=6$ in all experiments. Each of the $K$ channels is made available at an MC with probability $\mathrm{Pa}$. On the other hand, all the channels are always assumed available at the MR.

a) Intra-group assistance: Figure 2 shows the gain of using intra-group assistance in a single multicast group. The gain is defined as the percentage reduction in the multicast period of the unassisted multicast achieved by using assisted multicast ( $\frac{\text { unassisted-assisted }}{\text { unassisted }} \times 100 \%$ ). The optimal solutions for the two cases of unassisted multicast and intra-group

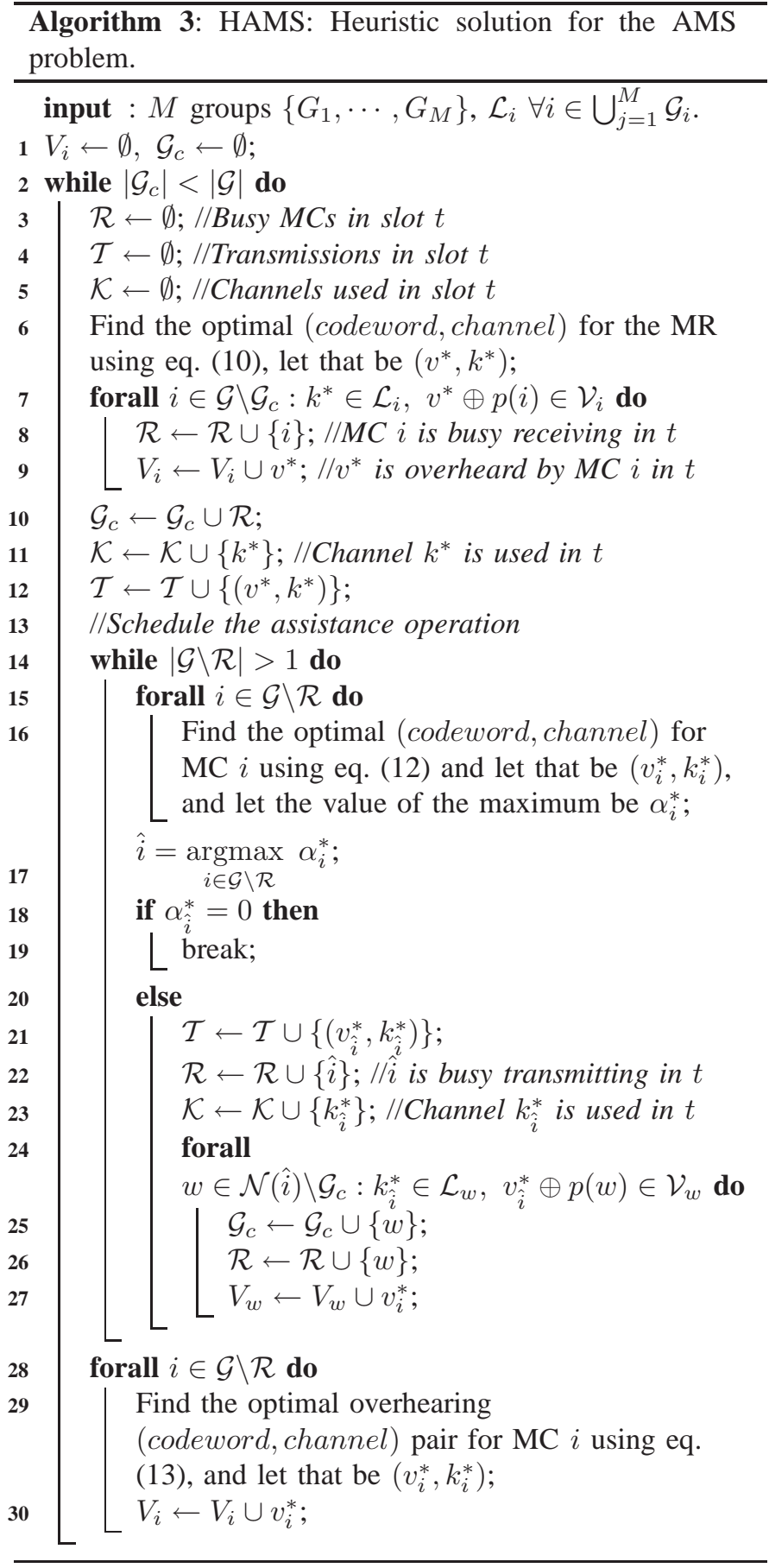

assisted multicast were obtained using the ILPs in Algorithms 1 and 2 respectively. We also evaluated the gain of intra-group assisted multicast by scheduling the problem using the HAMS algorithm. Each point is the figure is the average over a 100 randomly generated topologies. As the figure shows, the intragroup assistance achieves a significant gain over the unassisted case that increases with increasing the group size. On the other hand, the HAMS algorithm is performing well by achieving a considerable gain and being always within, on average, one time slot of the optimal solution obtained by Algorithm 2. In fact, HAMS was, on average, $\approx 0.63$ slots higher than the optimal assisted multicast schedule obtained using Algorithm 


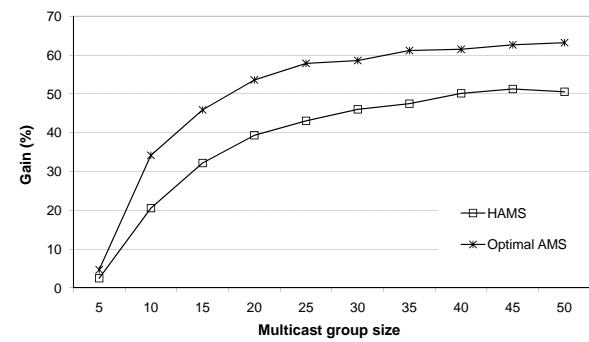

Fig. 2. The gain of using intra-group assistance in a single multicast group $(P a=0.25)$.

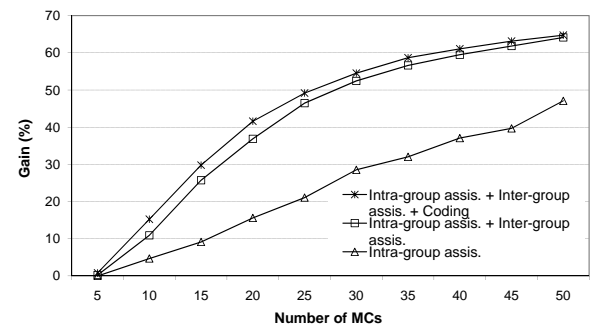

Fig. 5. Average gain of assisted multicast using different levels of assistance $(M=5, P a=0.25)$

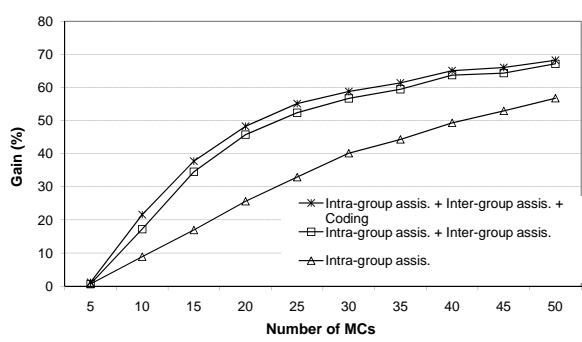

Fig. 3. Average gain of assisted multicast using different levels of assistance $(M=3, P a=0.25)$.

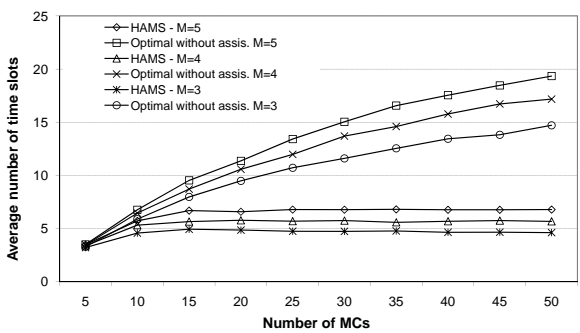

Fig. 6. Average multicast period with- and without-assistance $(M=3,4,5, P a=0.25)$.

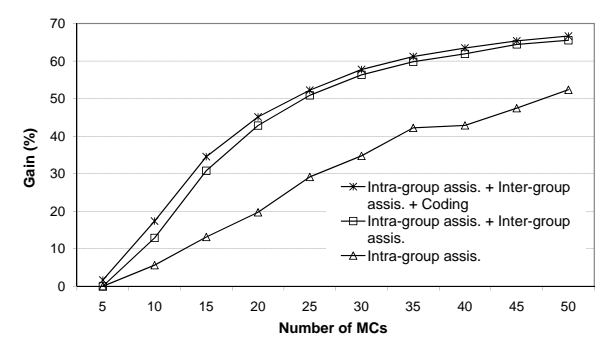

Fig. 4. Average gain of assisted multicast using different levels of assistance $(M=4, P a=0.25)$.

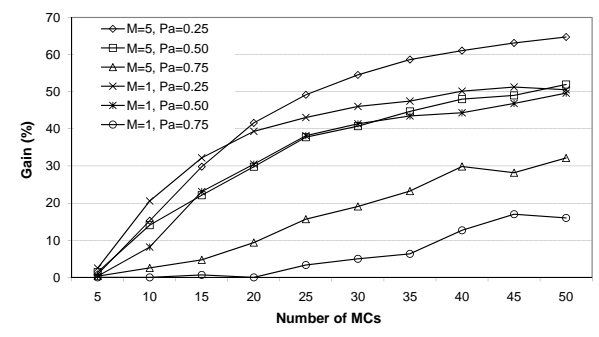

Fig. 7. The effect of channel availability on the gain of assisted multicast.
2 , and $\approx 2.11$ slots less than the optimal unassisted multicast schedule obtained using Algorithm 1.

b) Inter-group assistance: We now evaluate the benefit of using each of the three assistance operations: intra-group assistance, inter-group assistance, and the codeword exchange operation for multiple multicast groups. We vary the number of groups $M$ between 3, 4 and 5. For each case, we evaluate the gain using intra-group assistance only, intra- and intergroup assistance, and intra- and inter-group assistance with network coding. For the unassisted multicast case, we find the optimal schedule by running the ILP in Algorithm 1 for each one of the $M$ groups and summing up the optimal multicast periods for all individual groups to obtain the total multicast period. As for the assisted multicast scheduling, we used the HAMS algorithm. Figures 3, 4, and 5 correspond to the cases of $M=3,4$, and 5 respectively with each point in the figure be the average over a 100 randomly generated topologies. As the figures show, each level of assistance achieves some extra gain in the total multicast period. However, it is apparent that inter-group assistance has more influence on the total gain than the codeword exchange operation, yet the codeword exchange operation can still improve the scheduling performance. Figure 6 shows the actual averages of the multicast period for the data presented in Figures 3, 4, and 5.

c) The effect of channel availability: To understand the effect of channel availability on the achievable gain of the assisted multicast, we varied $P a$ between $0.25,0.50$, and 0.75 , for the cases of $M=1$ and $M=5$. As Figure 7 shows, the gain drops with higher channel availability. This is attributed to the fact that with high probability of channel availability, the expected number of MCs to be served in each slot is higher (more nodes share the same channel). This leaves less room for assistance causing the achievable gain to drop.

\section{CONCLUSIONS}

In this work, we have studied the problem of assisted multicast scheduling in wireless cognitive mesh networks. We have proposed an assistance paradigm that relies on receiver nodes to forward the multicast data to other receivers that have not yet received their own data. Furthermore, network coding was also proposed as another assistance technique that further reduced the total multicast period. Results show that the proposed assistance paradigm achieves a significant gain in reducing the total multicast period, i.e., overall throughput.

\section{REFERENCES}

[1] Joseph Mitola. An Integrated Agent Architecture for Software Defined Radio. PhD thesis, Royal Institute of Technology (KTH), Stockholm, Sweden, May 2000.

[2] Ian F. Akyildiz, Won-Yeol Lee, Mehmet C. Vuran, and Shantidev Mohanty. Next generation/dynamic spectrum access/cognitive radio wireless networks: a survey. Comput. Netw., 50(13):2127-2159, 2006.

[3] J. Unnikrishnan and V.V. Veeravalli. Cooperative spectrum sensing and detection for cognitive radio. In IEEE Global Telecommunications Conference (GLOBECOM) 2007., pages 2972-2976, November 2007.

[4] R. Thobaben and E. G. Larsson. Sensor-network-aided cognitive radio: On the optimal receiver for estimate-and-forward protocols applied to the relay channel. In Conference Record of the Forty-First Asilomar Conference on Signals, Systems and Computers (ACSSC) 2007, pages 777-781, November 2007.

[5] B. Mercier, V. Fodor, et al. Sensor networks for cognitive radio: Theory and system design. ICT Mobile Summit, June 2008.

[6] K.R. Chowdhury and I.F. Akyildiz. Cognitive wireless mesh networks with dynamic spectrum access. Selected Areas in Communications, IEEE Journal on, 26(1):168-181, Jan. 2008.

[7] Christina Fragouli, Jean-Yves Le Boudec, and Jörg Widmer. Network coding: an instant primer. SIGCOMM Comput. Commun. Rev., 36(1):6368, 2006.

[8] M. Gerla J.-S. Park W. Kim, S. Oh. Cocast: Multicast mobile ad hoc networks using cognitive radio. IEEE MILCOM 2009.

[9] Donglin Hu, Shiwen Mao, and J.H. Reed. On video multicast in cognitive radio networks. In IEEE INFOCOM 2009, pages 2222-2230.

[10] V. Fodor, I. Glaropoulos, and L. Pescosolido. Detecting low-power primary signals via distributed sensing to support opportunistic spectrum access. IEEE International Conference on Communications (ICC), 2009.

[11] SENDORA project. http://www.sendora.eu. 